\title{
Editorial
}

\section{Prospects of Artificial Light Harvesting Systems: An Introduction}

\author{
Jasper Knoester ${ }^{1}$ and Siegfried Daehne ${ }^{2}$ \\ ${ }^{1}$ Institute for Theoretical Physics and Materials Science Centre, University of Groningen, Nijenborgh 4, \\ 9747 Groningen, AG, The Netherlands \\ ${ }^{2}$ Scientific Consultation Siegfried Daehne, Kastanienallee 6, 12587 Berlin, Germany
}

Received 26 December 2006; Accepted 31 December 2006

Copyright (๑) 2006 J. Knoester and S. Daehne. This is an open access article distributed under the Creative Commons Attribution License, which permits unrestricted use, distribution, and reproduction in any medium, provided the original work is properly cited.

\section{INTRODUCTION}

The wonder of nature using visible light to synthesize countless organic compounds whose enthalpy of formation needs much higher energies than provided by sunlight partly has been unveiled when the structure of the so-called light harvesting complexes (LHCs) of some photosynthetic bacteria was solved by X-ray structural analyses in the nineties of the last century. Since then the dream of mankind to mimick natural photosynthesis of plants and bacteria seemed to be much more promising than ever before.

Former attempts to realize photosynthesis through accumulation of visible light quanta were based on the interaction of solid semiconductors with light absorbing dye molecules. In this way effective solar cells have been developed, but the chemical yield of such heterogeneous phases is basically limited by the size of the semiconductors' surface. Nevertheless, the oldest known artificial light harvesting system, namely, the formation of metallic silver clusters in photographic silver halide emulsions by reduction of silver ions through day-light exposure contributed much to the understanding of photosynthesis induced by dyes, the socalled spectral sensitization. It turned out, for instance, that the photoinduced electron and/or hole-transfer mechanisms strongly depend on the dyes' reduction and oxidation potentials in both their ground and excited states which can be varied over a range of more than one electron volt in dependence on the dyes' molecular structure. Another unique property of such spectral sensitizers is their aggregation tendency to form supramolecules, the so-called J-aggregates, which transport light energy (excitons) within a few picoseconds over many molecules at any place where it is needed to perform electron transfer process similar to natural LHCs (cf. the review of
T. Tani et al. in this special issue). Therefore, further research has to be done, for example, to improve the J-aggregates' effectivity through employment of self-assembled multilayer techniques (cf. the paper of A. Lodi et al. in this special issue).

In order to increase the chemical yield in artificial LHCs recent developments are aiming for photosynthesizing systems based on homogeneous phases. Especially synthetic variations of the structure of the natural LHCs' basic chromophore, the porphyrin skeleton (cf. the paper of H. Tamiaki et al. in this special issue), as well as syntheses of treelike dendrimers with covalently linked dye molecules, was promising. However, in the first example, the synthetic efforts are rather high and the variability of the chromophore itself and its redox potentials is low, and in the second case, the mutual arrangement of the dye molecules cannot be controlled, and thus, exciton migration is usually limited to incoherent energy-hopping processes; whereas coherent exciton propagation associated with highly ordered molecular arrangements takes place only by chance.

Recently, a breakthrough in developing artificial light harvesting systems has been achieved through combining the self-organization tendency of amphiphilic organic surfactants with the unique property of J-aggregates having coherent exciton migration similar to the natural LHCs. In this way, supramolecular particles, like nanotubes, molecular strands, and helices based on dye molecules, can be designed. The Jaggregation tendency of such amphiphilic dyes is so strong that in most cases coherent exciton migration is maintained even when additional molecules, like alcohols, surfactants, or even strong electron donors, are either intercalated in the supramolecular entities or chemically linked at the constituent amphiphilic dye molecules. Thus development and 
modeling of new supramolecular architectures on the basis of amphiphilic J-aggregates resembling the natural LHCs become possible. The qualitatively new properties of such particles indeed justifiy their new designation "Amphipipes" (cf. the review of S. Kirstein and S. Daehne in this special issue).

Consequently, the new experimental results also requires comprehensive extension of the theory of Frenkel excitons to cylindrical and helical molecular assemblies with the ability of exciton migration. Although the properties of Frenkel excitons in such aggregates resemble those of the well-known linear J-aggregates, they also reveal several novel aspects (cf. the review of J. Knoester in this special issue).

It is not surprising that amphipipes conceal many hitherto unknown properties. For instance, the double-wall structure of their nanotubes gives rise to new optical response and dynamics like interesting intrawall energy transfer processes which are strongly dependent on both the molecules' substituents and (or) the liquid environment (cf. the paper of A. Pugzlys et al. in this special issue). Also exciton-exciton annihilation in the nanotubes behaves quite unexpected. In contrast to the usual disappearance of exciton-exciton annihilation at low temperature owing to freezing thermal mobility, it takes place in amphipipes even at very low temperatures (cf. the paper by C. Spitz et al. in this special issue).

Also first photoinduced electron transfer reactions have been realized by amphipipes. For instance, amphipipe helices spectrally sensitize the photochemical reduction of noble metal ions in aqueous solutions by visible light yielding noble metal clusters. In principle this photoinduced reaction is analogous to the growth of metallic silver specks in spectrally sensitized photographic silver halide emulsions after light exposure. However, new questions arise then about the migration mechanism of the metal ions or photoelectrons in amphipipes when no semiconductor is present, as well as about the regeneration mechanism of the dye positive holes. (cf. the paper by S. Kirstein et al. in this special issue).

\section{FUTURE PROSPECTS}

Based on these results which were mostly presented at the SOLAR'06 Conference held at Cairo in January 2006, there is no doubt that amphipipes are opening fascinating new avenues to mimic natural LHCs in homogeneous phases. Nevertheless, only a tiny door slit has been disclosed so far. Thus an immense work will have to be done in future, for instance, the following.

(i) The hitherto known amphipipes are derivatives from one and the same chromophore whose structural modifications are indispensible for further progress, such as lengthening and/or substitutions of the dyes' polymethine chain, symmetrical and asymmetrical alterations of their heterocyclic residues, variations of their hydrophobic groups, and changing the acid or basic nature of their hydrophilic substituents. For instance, hydrophilic phosphatoalkyl substituents might be applied in view of generation of DNA-like double helices. Most important will be similar variations of the chromophores themselves. Owing to their widely varying reduction and oxidation potentials, it will be possible to select the most favourable amphipipe for a certain photochemical reaction in order to optimally adjust the chemical reaction potential needed.

(ii) As the morphology of the amphipipe nanostructures strongly depends on their microenvironmental conditions, such as the presence of hydroxylic compounds, of different surfactants, and presumably of proteins, the number of possible amphipipe architectures still to be brought to light will raise exponentially when such compounds are intercalated in amphipipes. Furthermore, it will also be possible to intercalate photochemical reaction partners themselves, for example, strong electron donors or acceptors, like the viologens, into amphipipes for in situ photochemical reactions without interrupting their characteristic coherent exciton delocalization. Even intercalation of dye molecules covalently bound to certain reaction partners seems to be conceivable in order to design artificial LHCs closest to the natural ones.

(iii) Up to now it has only been demonstrated for one example that the coherent exciton delocalization in an amphipipe helix extends over some ten molecules at room temperature, and over some hundred molecules at low temperature within few picoseconds. However, apart from the already mentioned results from Pugzlys et al. who merely compared two amphipipes having one and the same chromophore but different hydrophilic substituents, nothing is known about the dependence of the optical response and exciton dynamics on the amphipipes manifold morphologies nor on the structure of their constituent molecules.

(iv) After first photoinduced electron transfer reactions have been realized through the already mentioned photoreduction of noble metal ions in the presence of amphipipe helices, it will be imperative next to investigate the nearly boundless number of photochemical reactions having reaction energies in the UV region which might be made possible by light harvesting of sun light through amphipipes.

(v) Finally, the challenging sideway will have to be explored, that chiral amphipipe helices are spontaneously generated from achiral dye molecules. So far it is known that in aqueous solutions of dye monomers exists a thermodynamical equilibrium between several conformers owing to interactions of the dyes' hydrophobic substituents as well as the formation of weak intramolecular hydrogen bonds between the dyes' hydrophilic substituents. Obviously, some of the molecules are temporarily twisted necessarily causing chirality which is permanently stabilized when two twisted molecules having by chance identical handedness form a dye dimer. Such a chiral dimer then acts as a template for the growth of a helix having the same handedness. Furthermore such helices might transfer their chirality to other dye dimers or the helices themselves might break into smaller parts before their growth continues. In this way each solution has a surplus of helices having equal handedness.

On repetition the experiments described an equal number of amphipipe solutions have to be expected, one of them containing preferably left-handed helices, and the other one preferably right-handed helices. It is a mystifying and to date unexplained result that the expected parity of examples could not be confirmed. In most cases left-handed helices were 
obtained. Possibly this result might provide the key to get deeper insight to the origin of asymmetry during the evolution of life.

The authors are quite confident that this special issue of the International Journal of Photoenergy will challenge broad new and successful activities in the field of amphiphilic J-aggregates which are most prospective candidates for realizing best suited artificial light harvesting systems.

Jasper Knoester Siegfried Daehne 




International Journal of

Medicinal Chemistry

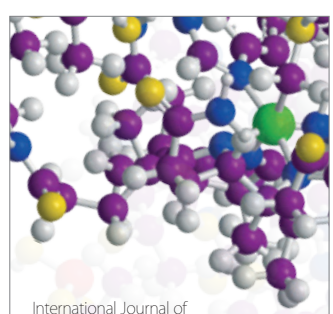

Carbohydrate Chemistry

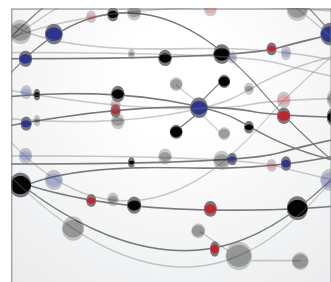

The Scientific World Journal
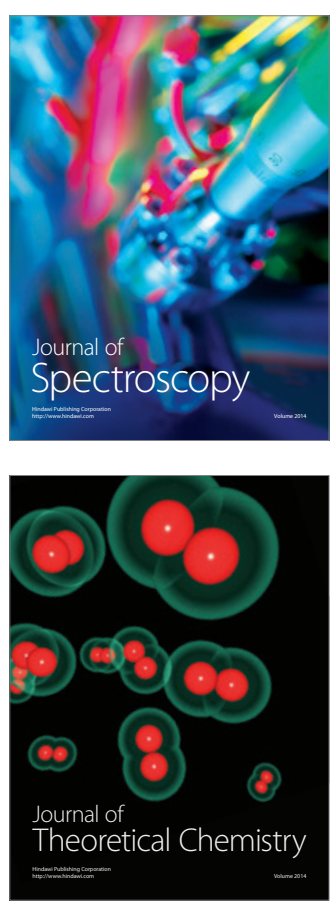
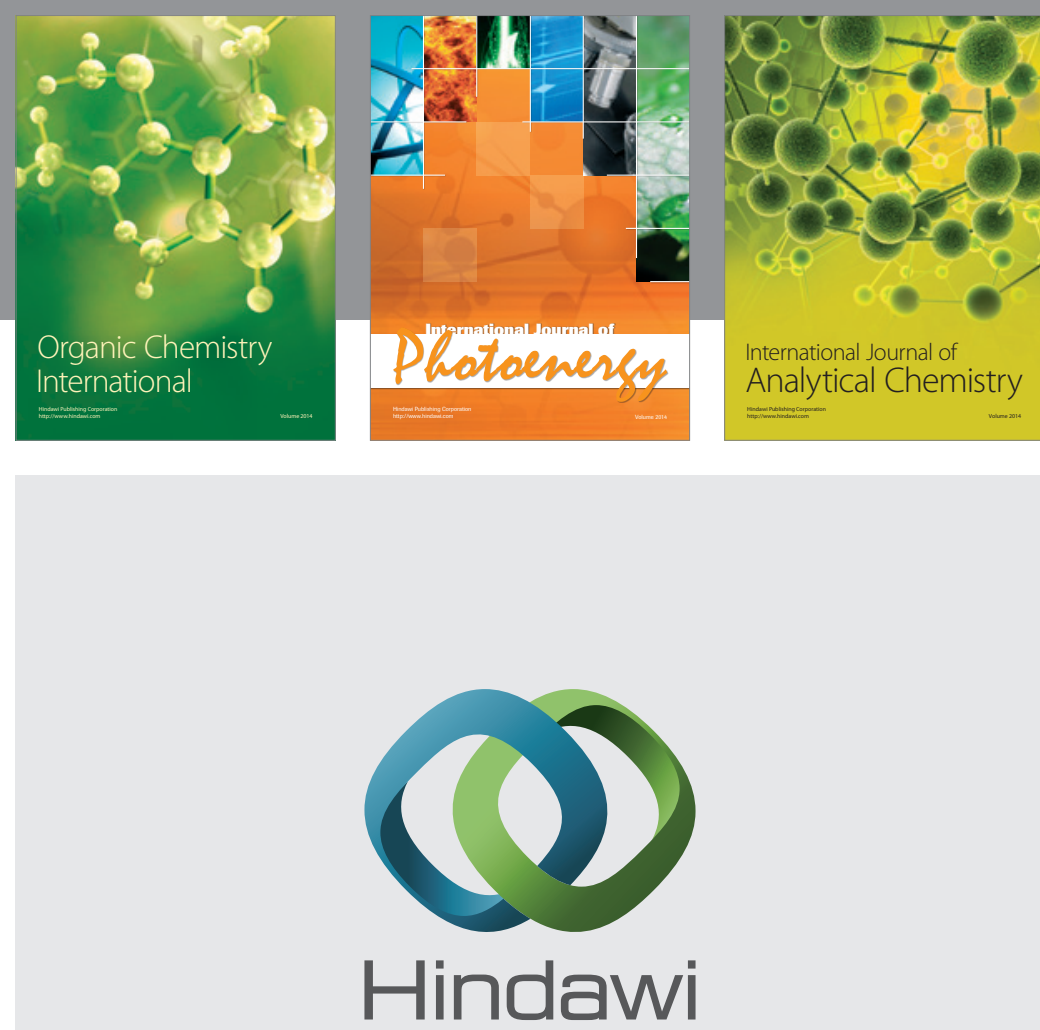

Submit your manuscripts at

http://www.hindawi.com
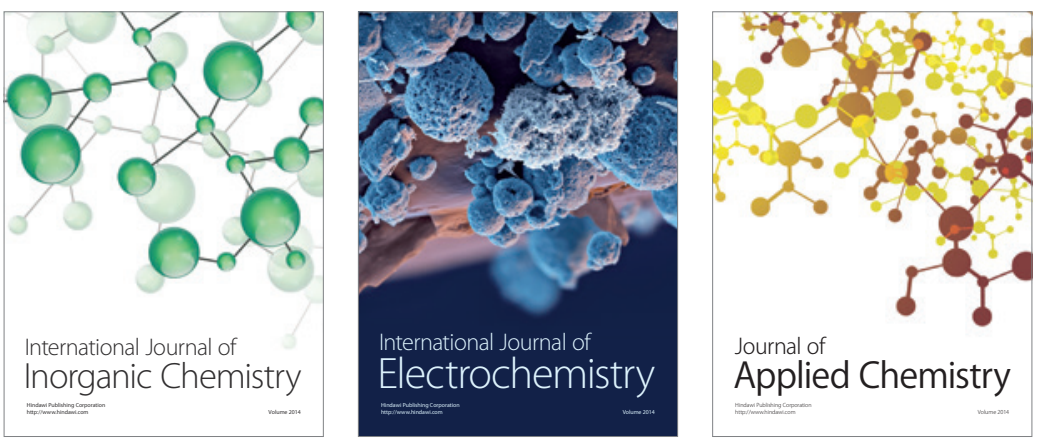

Journal of

Applied Chemistry
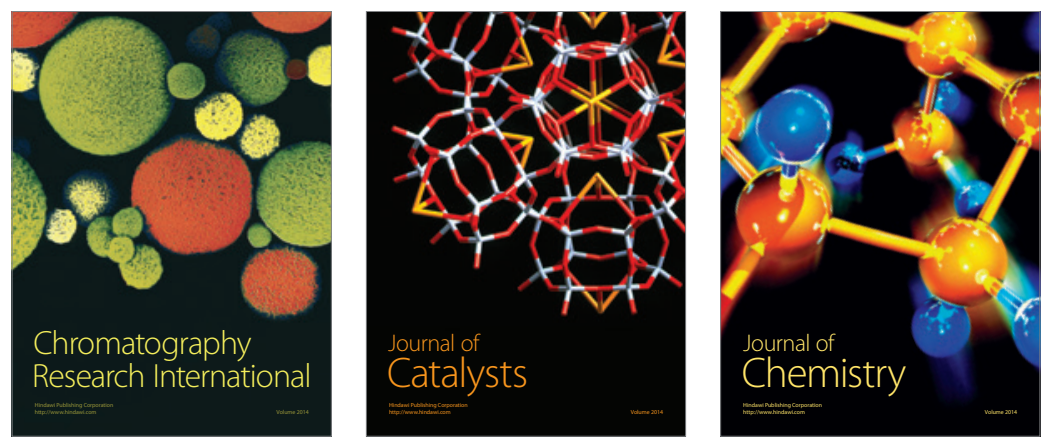
